\title{
lieuxdits \#15
}

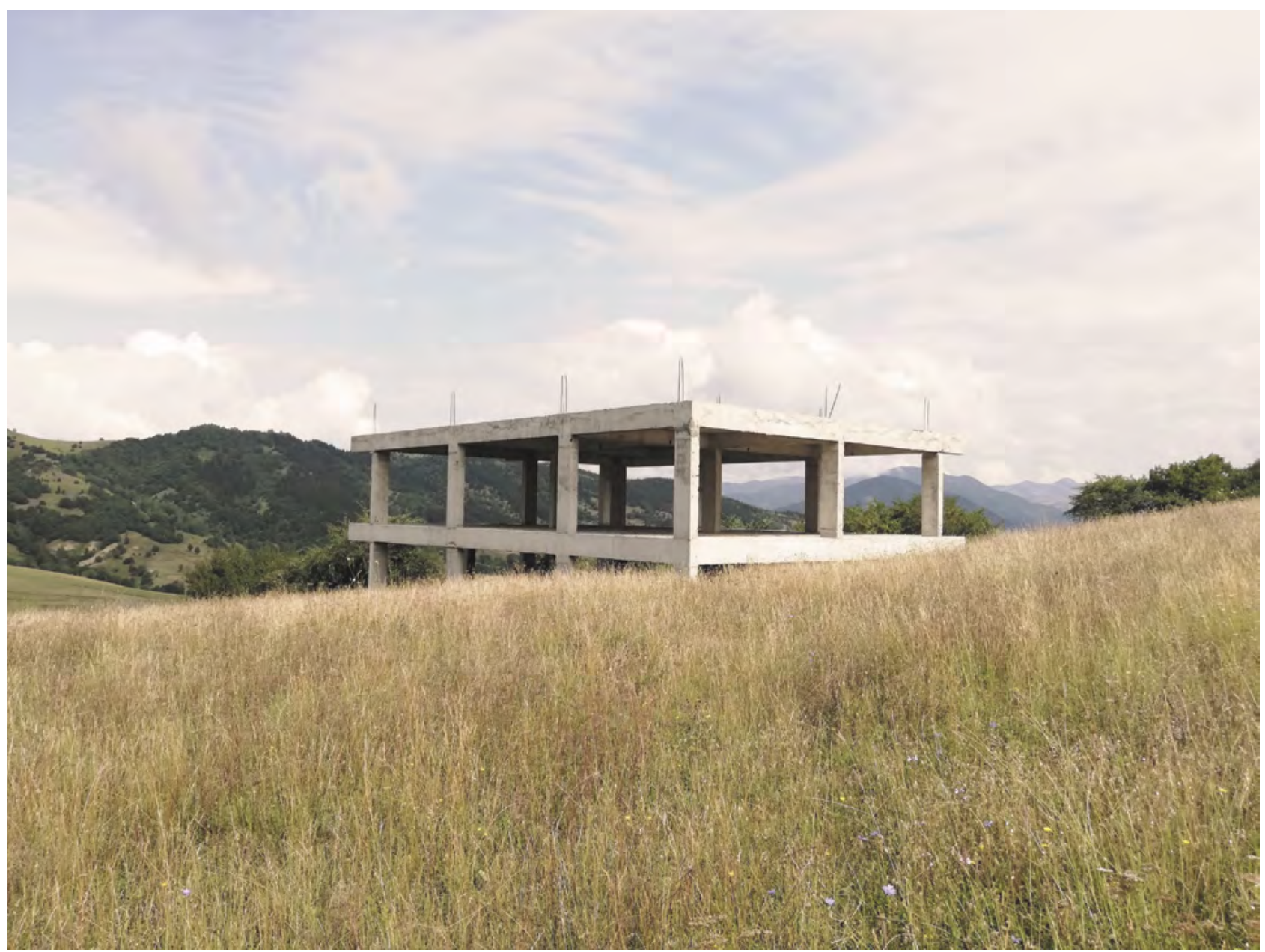

Faculté d'architecture, d'ingénierie architecturale, d'urbanisme de l'Université catholique de Louvain 


\section{Recyclage de l'urbain bruxellois}

Analyse typo-morphologique de la ressource : les tissus mixtes bruxellois

Barbara Le Fort

Bruxelles, comme d'autres métropoles post-industrielles européennes, a hérité d'un important parc immobilier industriel faisant l'objet de nombreux projets de reconversion. Si le déclin industriel bruxellois a sonné le glas de nombreuses activités manufacturières, il n'a pas pour autant créé un chancre urbain au cœur de la ville, dans la partie centrale de la zone du canal. En effet, en marge des reconversions lourdes des grandes manufactures iconiques du début du $x x^{e}$ siècle et de l'émergence des lofts et espaces de bureaux au design industriel, se trouvent des espaces productifs intégrés au tissu urbain résidentiel et hébergeant encore de nombreuses activités économiques de taille modeste. Ces dernières profitent de la combinaison de trois facteurs : le prix faible d'un foncier dévalorisé, la localisation centrale proche des quartiers les plus denses de la capitale et la diversité des superficies planchers des bâtiments généralement implantés en cœur d'îlot sur des parcelles originellement larges et profondes. Ces mêmes facteurs attirent aujourd'hui les acteurs de la rénovation urbaine voyant en ces parcelles des opportunités de projet pour "améliorer les quartiers" du "croissant pauvre" bruxellois.

Abordé sous l'angle spatial, qu'il soit en friche - un déchet résultant du déclin de l'activité industrielle - ou non, le bâti industriel en cœur d'îlot devient une ressource d'espace et de projet au cœur de la ville dense. Mais à ce titre, force est de constater le manque cruel d'une connaissance fine de cette ressource.

La recherche vise dès lors à produire le recensement et la description spatiale et typo-morphologique de la ressource : les tissus mixtes bruxellois. La cartographie inédite de ces tissus induit une nouvelle géographie bruxelloise. Les premiers résultats de cette cartographie offrent un éclairage nouveau sur le débat et les essais de production de la métropole mixte et productive.

\section{Tissus bâtis mixtes}

Les espaces semi-industriels présents dans la vallée industrielle bruxelloise sont intégrés au sein des îlots. Ces îlots urbains ont été progressivement saturés par des processus de densification, consolidant dans un même front de rue une juxtaposition de petites industries, des maisons de patrons et de modestes maisons d'ouvriers (Vandermotten, 2014). Un mélange fonctionnel de différentes typologies architecturales caractérise ces îlots, avec un très haut degré de saturation. Nous les appelons "tissus bâtis mixtes".

S'inscrivant dans la suite des recherches sur la typo-morphologie des tissus ur- bains bruxellois (Lacour et al., 1987 ; De Visscher, 2013 ; Ledent, 2014), cet article présente une exploration typo-morphologique des configurations bâties qui organisent des îlots mixtes. Notre étude considère les bâtiments et les parcelles comme des premiers éléments urbains. L'approche dézoome progressivement pour caractériser la structure qui lie les éléments urbains : le tissu urbain (Caniggia \& Maffei, 2000). Notre grille d'analyse pour les études de cas est construite sur la conjonction de trois composantes des tissus urbains : les configurations de parcelles, les configurations bâties et la depth configuration ou profondeur du front bâti (Panerai et al, 1999 ; Habraken, 2000 ; Clossick, 2017). Le front de rue est l'échelle de base pertinente pour l'analyse du tissu urbain (Porta \& Romice, 2010 ; Carmona, 2014).

\section{Trois conditions stratégiques des espaces semi-industriels en cœur d'îlot}

L'étude de cas "Quai de l'Industrie" est un exemple paradigmatique de la corrélation entre les formes de parcelles, les configurations bâties et l'organisation de la relation à la rue, les trois conditions stratégiques pour l'implantation et le maintien d'espaces semi-industriels en cœur d'îlot (figure1).

Le front de rue analysé fait partie d'un îlot mixte urbanisé entre 1868 et 1899 suite au développement industriel du quartier Heyvaert, de part et d'autre du nouveau canal de Charleroi (inauguré en 1832). Un tracé régulier de rues formant de grands îlots a été conçu à travers les parcelles agricoles à la demande des industriels afin de développer leurs activités. La trame qui en résulte semble répondre au besoin d'un environnement urbain équilibré mélangeant usines, entrepôts, petits ateliers, maisons de patrons et d'ouvriers et petits magasins de quartier. Le tracé des parcelles traduit une hétérogénéité typologique répondant à différentes conditions d'implantation. On observe alors dans le même front de rue : grandes implantations semi-industrielles, petits ateliers urbains, rez-de-chaussée commercial avec étages habités.

Sur des parcelles larges $(>9 \mathrm{~m})$ et importantes situées aux médianes du rectangle, on trouve des configurations bâties homogènes qui accueillent une typologie architecturale industrielle tous les bâtiments sont exclusivement conçus pour accueillir une activité productive, logistique ou économique, y compris les espaces administratifs avec une relation physique et visuelle directe de l'espace industriel à la rue. verts urbains privés - exemples Sources : TVBuONAIR 2017 CREAT-UCL 2017 / Ségolène Gréant 2017

3 L'outil dynamique (Réalisation: CREAT, 2017)

4 TVBuONAIR(Source: CREAT) 

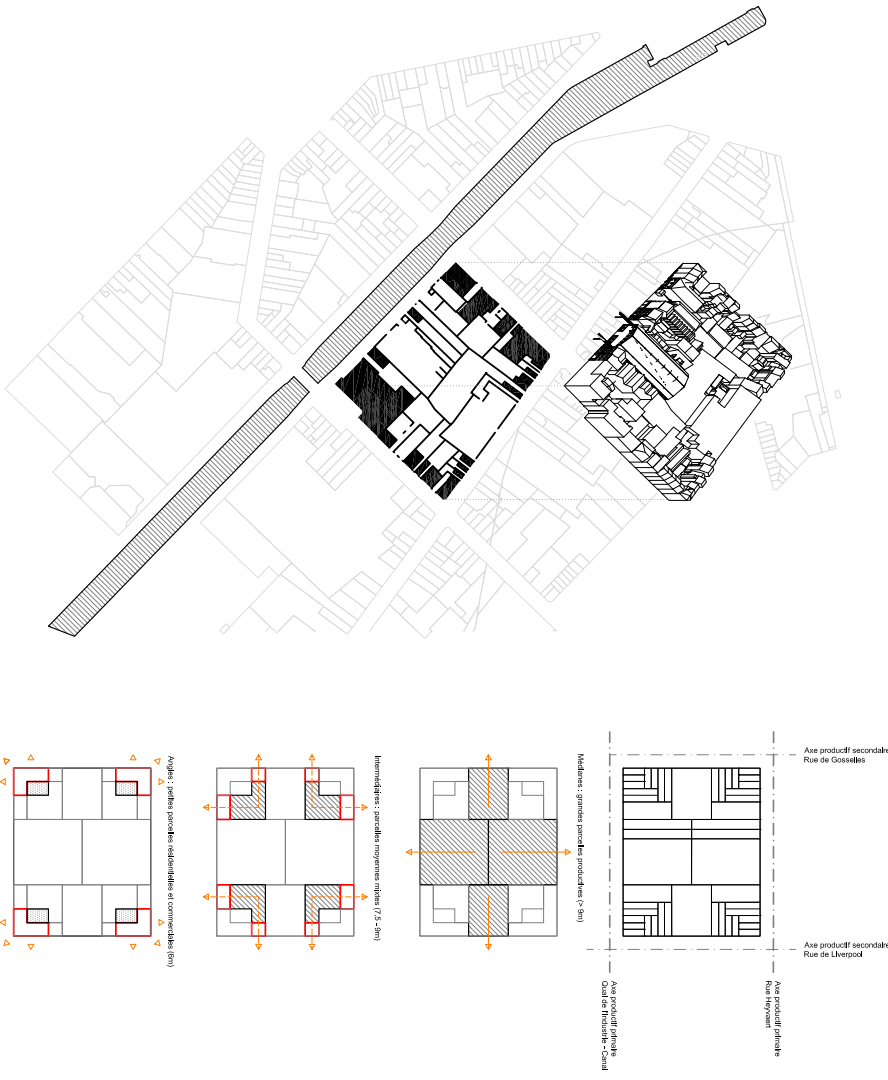

Homogène
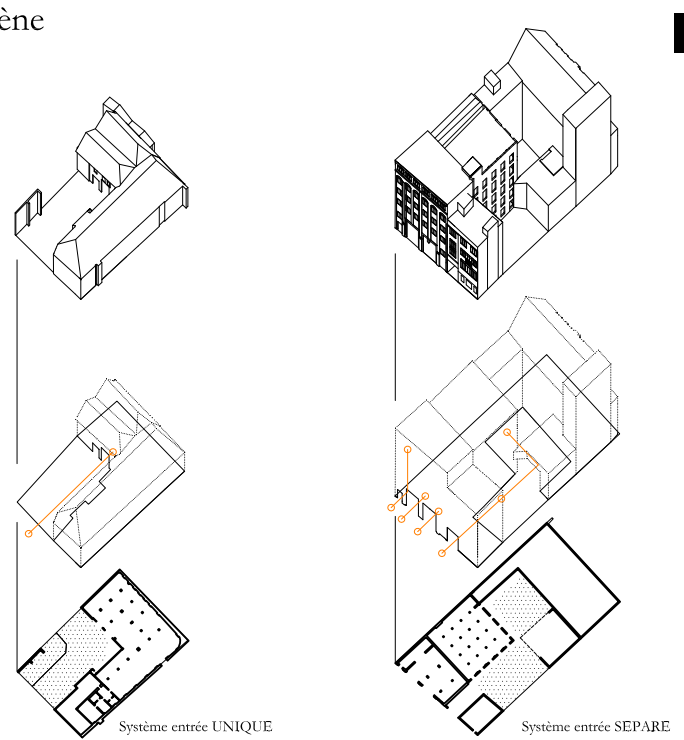

Mixte
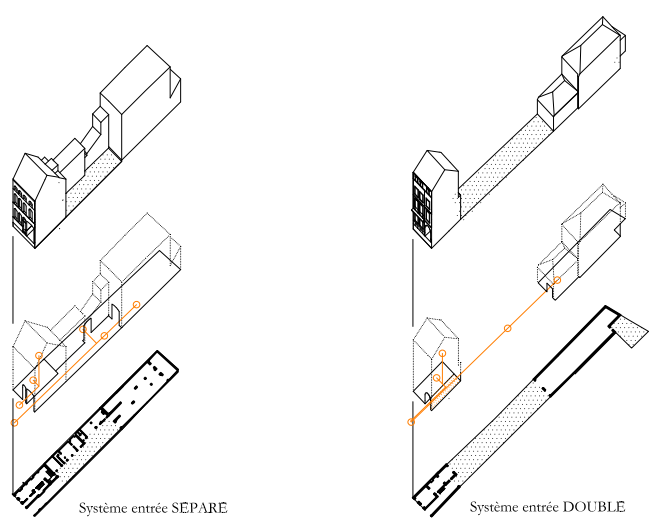

2
Entre la médiane et l'angle, on trouve des parcelles de taille intermédiaire (entre 7,5 et $9 \mathrm{~m}$ ) qui accueillent configurations bâties mixtes : un front bâti résidentiel, variante du modèle de la maison bruxelloise, avec une porte cochère ou de garage permettant l'accès à une cour, un entrepôt ou un atelier à l'arrière. Le rez-de-chaussée est parfois utilisé comme vitrine commerciale de l'activité installée à l'arrière.

Enfin, à l'angle de l'îlot on trouve des parcelles étroites $(6 \mathrm{~m})$ avec peu de profondeur. La situation compliquée de l'angle est résolue par l'implantation d'un magasin de proximité ou un horeca au rez-de-chaussée profitant de l'emplacement stratégique du carrefour.

La depth ou profondeur du front de rue qui permet l'accessibilité aux espaces productifs depuis la rue est traduite physiquement et spatialement en trois système-entrées (figure 2). Dans la configuration homogène productive, le système-entrée unique présente l'interface d'un seul type d'espace, un entrepôt par exemple. Le système-entrée séparé, que l'on trouve aussi dans les configurations mixtes, dissocie les entrées logistiques - accès appropriés pour les véhicules à moteur vers les espaces productifs et de stockage - des espaces administratifs ou domestiques - accès piéton aux espaces de bureaux ou aux espaces résidentiels. Enfin, le système-entrée double intègre à la fois l'accès logistique, administratif et domestique via une seule entrée. Dans cette configuration, l'accès est partagé entre les utilisateurs d'espaces résidentiels côté rue et les utilisateurs d'espaces semi-industriels à l'arrière.

Dans la construction de configurations mixtes, la profondeur permet un fort potentiel relationnel entre l'espace public et le cœur d'îlot semi-industriel. Ce potentiel relationnel, combiné au mélange de configurations parcellaire et de typologie architecturale, contribue à l'intensité des tissus urbains mixtes. Parce qu'elles permettent un mélange fonctionnel et des adaptations, ces morphologies mixtes fonctionnent comme des métabolismes résilients (Clossick, 2017 ; Feliciotti, 2017).

\section{Matrice typo-morphologique}

L'établissement d'une typologie des configurations semi-industrielles apparaît plus que nécessaire pour cartographier et étudier plus globalement les tissus semi-industriels bruxellois et rendre compte de cette variation. La configuration bâtie ainsi que les systèmes-entrée associé au mode d'occupation de la parcelle par le bâti - saturé, sur cour, en recul ou ouvert - sont les trois critères qui définissent les types (figure 3). Ce dernier critère offre deux 
Homogène

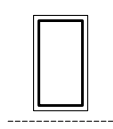

Saturé

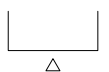

Unique

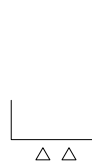

Séparé

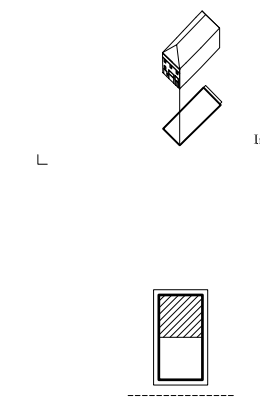

Saturé

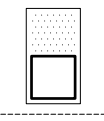

Cour

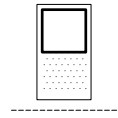

Recul

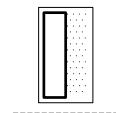

Ouvert

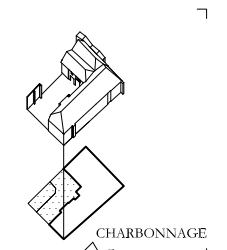

$+$
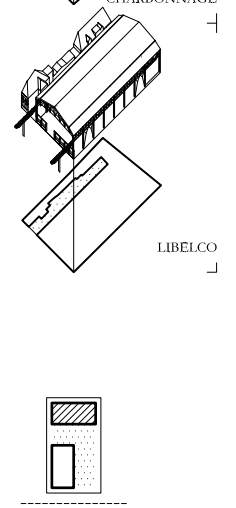

Ouvert

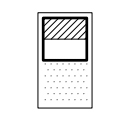

Recul
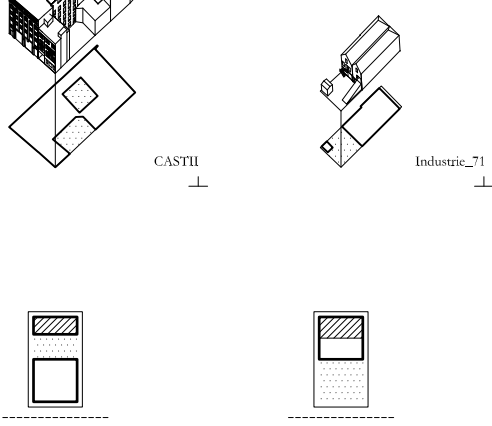

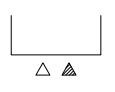

Séparé

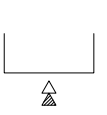

Double

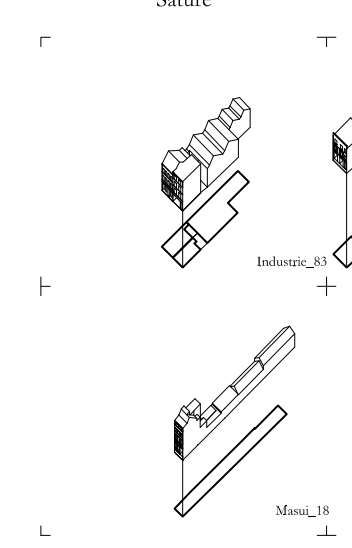

informations. D'une part il indique la part de saturation de la parcelle et donc la possibilité d'une densification ou le besoin d'une dédensification. D'autre part, il donne une indication sur l'activation de l'espace public par le front bâti qu'il constitue : les implantations saturées et sur cour présentant un front bâti fermé (dans un contexte bruxellois de tissu consolidé de maisons mitoyennes) à l'inverse des implantations en recul et ouvertes.

\section{Carte des configurations mixtes, premiers résultats}

La matrice typo-morphologique permet de donner à chaque parcelle étudiée une valeur. La cartographie qui en résulte nous permet de montrer la complexité du tissu construit mixte et ses différentes échelles pertinentes. Elle met en valeur les différents modes de coexistence morphologique entre typologies productives, mixtes, commerciales et "non productives" (résidentiel/bureaux/ aménités) (figure 4). Par exemple pour le quartier Heyvaert (Cureghem), nous observons des îlots carrés mixtes présentant la même distribution de configurations productives que le cas d'étude présenté ci-dessus. Les petits nœuds commerciaux apparaissent clairement aux carrefours des rues (parcelles commerciales d'angle). La Chaussée de Ninove, la Chaussée de Mons et la rue Ropsy-Chaudron émergent comme axes commerciaux.

La dimension de l'îlot, et en particulier sa profondeur, ainsi que la largeur de parcelles sont deux déterminants fondamentaux de l'implantation d'activité dans le tissu. En effet, plus l'îlot est important, plus il pourra accueillir des parcelles larges et profondes permettant l'implantation d'activité productive : des configurations mixtes sur des parcelles entre $7,5 \mathrm{~m}$ (système-entrée double) et $9 \mathrm{~m}$ de large (système-entrée séparé) et généralement des configurations homogènes sur des parcelles de plus de $9 \mathrm{~m}$ de large. À l'inverse plus l'îlot est petit et étroit plus il accueillera des implantations résidentielles (parcelles de $6 \mathrm{~m}$ de large).
Le Canal et la rue Heyvaert sont les deux axes productifs primaires le long desquels s'installent les implantations produc tives les plus importantes. Les rues de Gosselies et Liverpool présentent un front bâti mixte (couronne d'îlot résidentiel et cœur productif)

2

Deux typologies bâties permettent l'implantation d'activité productive en cœur d'îlot : homogène productif, ou mixte. La relation à la rue, ou depth, se fait via trois systèmes-entrée : unique, séparé ou double.

3 Matrice typo-morphologique constituée à partir de trois critères : la configuration bàtie, le système-entrée et le mode d'occupation de la parcelle (en construction). 
Carte des configurations mixtes, zoom sur le quartier Heyvaert (en construction, situation juin 2018)

\section{Références bibliographiques}

CANIGGIA, G., \& MAFFEI, G. L. (2000). Composition architecturale et typologie du bâti : 1. lecture du bâti de base. Versailles: Ville Recherche Diffusion. CARMONA, M. (2014). London's Local High Streets: The Problems, Potential and Complexities of Mixed Street Corridors, Progress in Planning, 100 (August 2015): 1-84. CLOSSICK,J. (2017). The depth structure of a London high street: a study in urban order, $\mathrm{PhD}$ thesis, London Metropolitan University DE VISSCHER,J.P. (2013). Indivision, thèse de doctorat Université catholique de Louvain, Prom.: Stillemans, Jean, Louvain-la-Neuve FELICIOTTI, A. (2017). “Urban regeneration, masterplans and resilience: the case of Gorbals, Glasgow", Urban Morphology, n²1(1), International Seminar on Urban Form, France, 2017, pp. 61-79 HABRAKEN, N. J., \& TEICHER, J. (2000). The Structure of the Ordinary : Form and Control in the Built Environment. Cambridge, Mass.: MIT Press.

LACOUR, M., DELHAYE, I., DUMONT,M. (1987). Morphologie urbaine à Bruxelles, Centre d'étude, de recherche et d'action en architecture, Bruxelles LEDENT, G. (2014). Potentiels relationnels : l'aptitude des dispositifs physiques de l'habitat à soutenir la sociabilité: Bruxelles, le cas des immeubles élevés et isolés de logement, thèse de doctorat Université catholique de Louvain, Prom. : Olivier Masson, Louvain-la-Neuve PANERAI, P., DEPAULE,J-C., DEMORGON, M. (1999). Analyse urbaine, éditions Parenthèses,

France.

PORTA, S., ROMICE, O. (2010).

Plot-Based Urbanism: Towards

Time-Consciousness in Place-

Making, working paper, UDSU, University of Strathclyde, Dept. of Architecture,

VANDERMOTTEN, C. (2014). Bruxelles, une lecture de la ville : de l'Europe des marchands à la capitale de l'Europe. Bruxelles: Éd. de l'Université de Bruxelles.

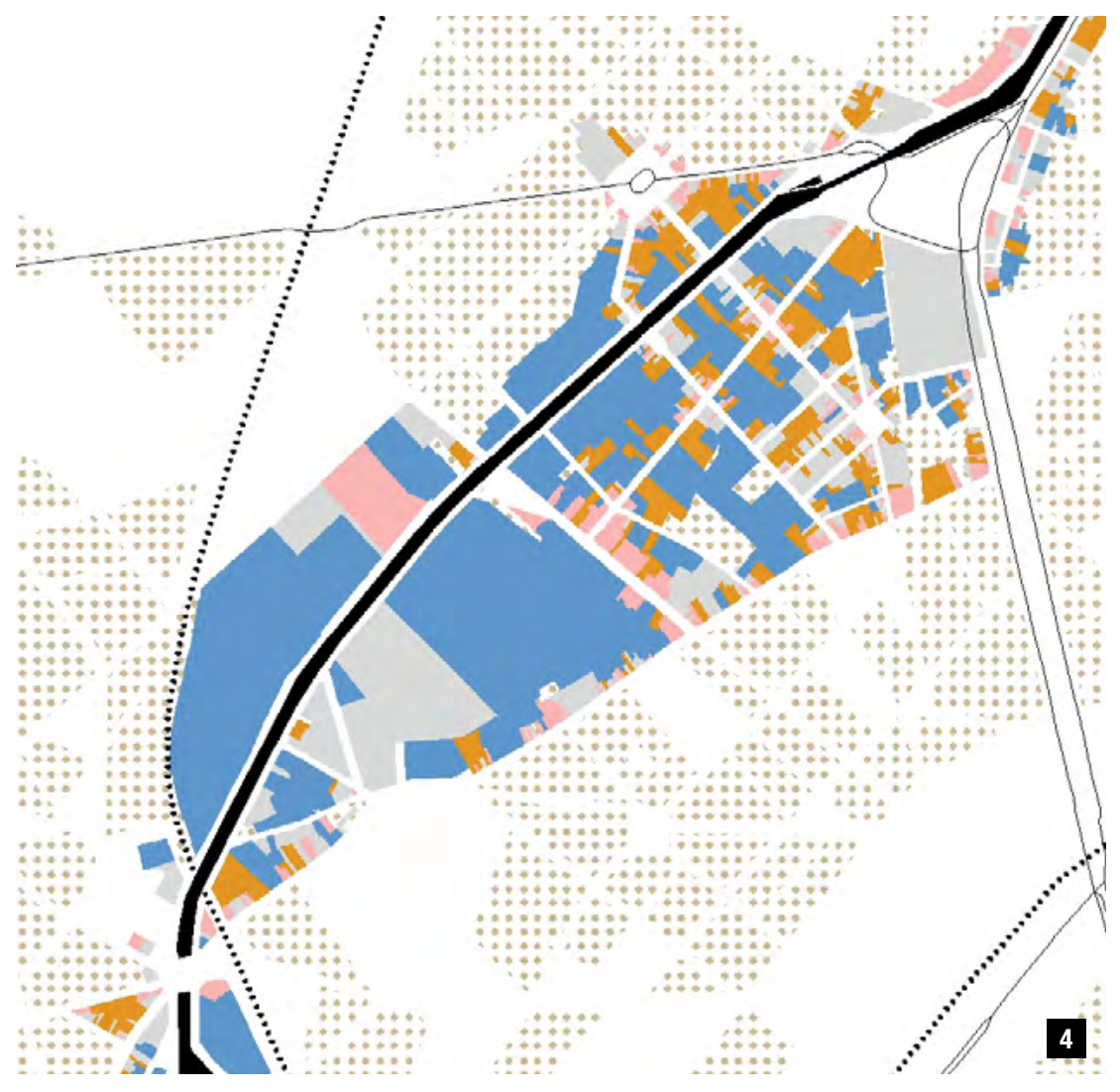

Heyvaert

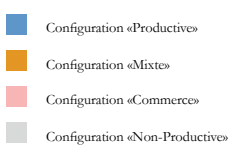

\section{Conclusion}

Par l'angle typo-mophologique, cette recherche apporte de nouvelles connaissances pour définir le tissu urbain mixte à différentes échelles. Les premiers résultats plaident en faveur d'une analyse et de représentations multi-échelles pour caractériser les tissus urbains mixtes bruxellois. En effet, on observe un mélange typologique à l'échelle du bâtiment, à l'échelle du terrain, à l'échelle de la rue et enfin à l'échelle du quartier. Ces différentes échelles doivent être prises en compte dans les projets d'urbanisme qui envisagent la protection et le renforcement de la diversité fonctionnelle. De plus, pour envisager des configurations mixtes, les urbanistes bruxellois doivent cesser de penser le territoire bruxellois en termes d'îlots urbains mais bien en termes système rue-front bâti. 